
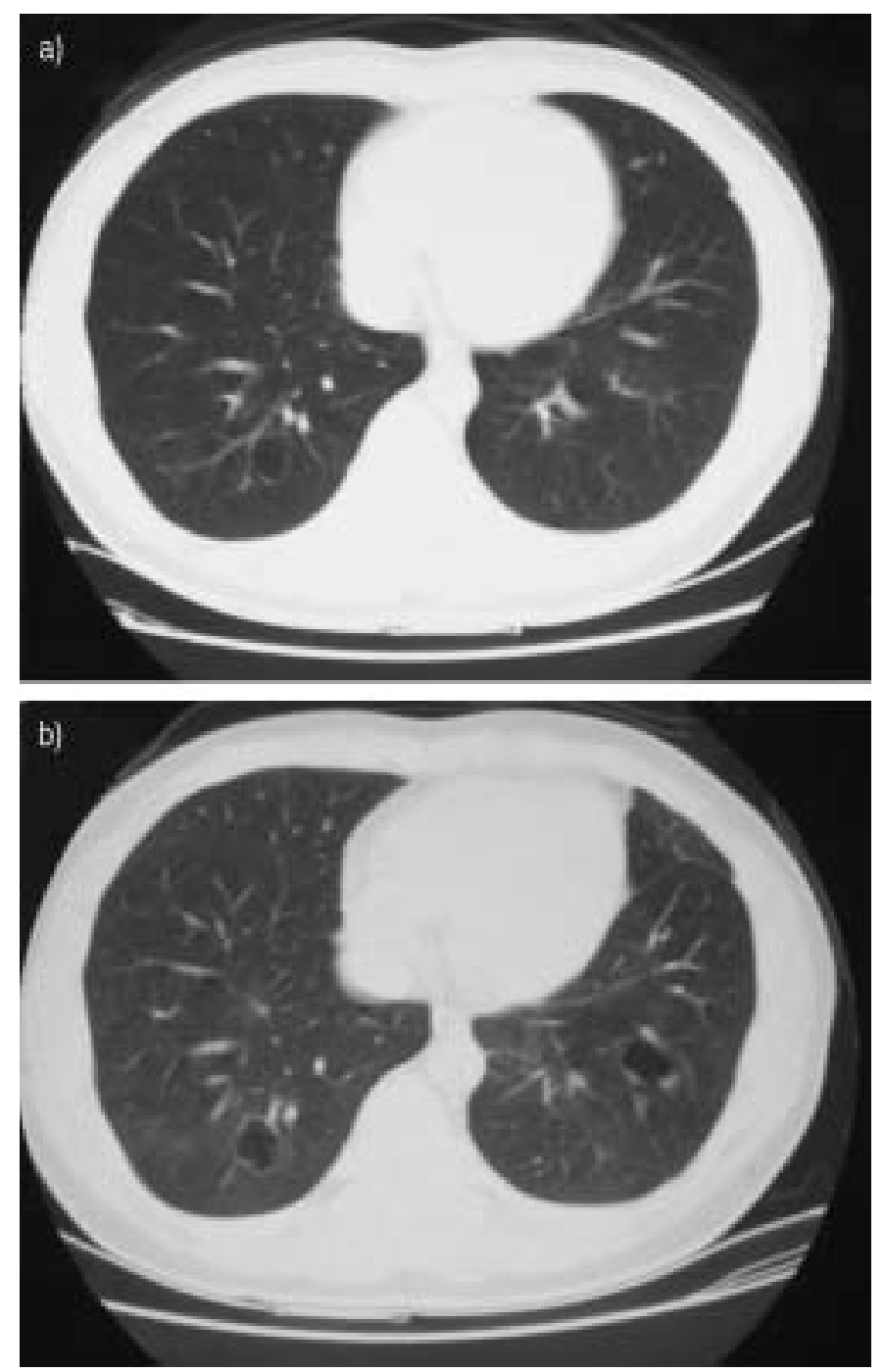

FIGURE 1. Computed tomography scan of the patient showing: a) an emerging thin-walled cystic lesion in the right lower lobe; and b) a slight increase in size of the original right lesion and a new development in the contralateral side at 2-yr follow-up.

tomography scan revealed bilateral multiple cystic lesions with thin smooth walls of varying sizes. However, the male sex prompted us to exclude a more sinister cause for the lesions
[2], and upon further questioning the patient had complained of right thigh pain for several weeks. Examination revealed a fixed soft tissue mass in the mid-thigh. Histology from the thigh mass biopsy and bilateral video-assisted thoracic surgery bullectomy [3] confirmed pulmonary epithelioid sarcoma metastases. Subsequent investigations showed no other organ was involved. The pulmonary lesions progressed over a 5-yr period, eventually leading to respiratory failure.

Pulmonary cysts as the sole metastatic manifestation of sarcomas are rare, with $<20$ cases reported [4]. The cysts are usually thin walled, without debris and slow growing (fig. 1).

Typically, the cystic lesions are not evident on chest radiographs, but are well visualised on computed tomography scans, where they may mimic benign bullous disease. The diagnosis may not be established until patients present with pneumothorax necessitating surgical intervention and biopsy $[3,5]$.

Clinicians should be aware of this important differential diagnosis of bilateral pulmonary cystic lesions, and exclude metastatic sarcoma in the assessment of their patients.

C.S.H. Ng, S. Wan, T.W. Lee and A.P.C. Yim

The Chinese University of Hong Kong, Hong Kong.

\section{REFERENCES}

1 Battistini E, Gambini C, Rossi UG, et al. Spontaneous pneumothorax in a 24-year-old female. Eur Respir J 2005; 25: 575-580.

2 Johnson SR, Whale CI, Hubbard RB, Lewis SA, Tattersfield AE. Survival and disease progression in UK patients with lymphangioleiomyomatosis. Thorax 2004; 59: 800-803.

3 Yim APC, Ng CSH. Thoracoscopic management of spontaneous pneumothorax. Curr Opin Pulm Med 2001; 7: 210-214.

4 Hasegawa S, Inui K, Kamakari K, Kotoura Y, Suzuki K, Fukumoto M. Pulmonary cysts as the sole metastatic manifestation of soft tissue sarcoma. Chest 1999; 116: 263-265.

5 Traweek T, Rotter AJ, Swartz W, Azumi N. Cystic pulmonary metastatic sarcoma. Cancer 1990; 65: 1805-1811.

\title{
Nontraumatic chylothorax and previous cancer: a role for positron emission tomography?
}

To the Editors:

Nontraumatic chylothorax in the adult is suggestive of a tumour, mainly lymphoma and metastatic carcinoma, particularly when prior neoplasm is diagnosed. If radiological procedures reveal no mediastinal lesion, surgical exploration may be suggested. Conversely, a tumour might become evident even 6-12 months after the appearance of chylothorax 
[1]. Positron emission tomography (PET) plays a critical role in the detection of numerous malignancies, including lymphoma and carcinoma, and even appears to be more sensitive for supradiaphragmatic disease involvement [2]. As I recently experienced, PET could be an excellent tool in the approach to chylothorax when conventional radiological procedures are inconclusive.

We report here a 59-yr-old female who was admitted on May 2004 because of bilateral pleural effusion. She had undergone a left mastectomy 14 yrs previously because of breast carcinoma. There was no other clinical condition in her life to comment on.

She had begun to have progressive thoracic discomfort 10 days prior to admission, which sometimes became more intense when swallowing. During the last $48 \mathrm{~h}$, she had developed mild dyspnoea on exertion.

On physical examination, the patient was afebrile and eupnoeic at rest. Her pulse was 80 and blood pressure was $130 / 80 \mathrm{mmHg}$. Oxygen saturation was $97 \%$ while the patient was breathing ambient air. Jugular venous pressure was not elevated. There were no palpable lymphadenopathies. Cardiac auscultation and abdominal exploration revealed no abnormality. On pulmonary auscultation, bilaterally decreased breath sounds were evident.

Thoracic radiograph and computed tomography (CT) of the thorax and abdomen demonstrated bilateral pleural effusion of moderate degree, without any other abnormality. Thoracentesis was performed and milky exudative pleural fluid ( $\mathrm{pH} 7.5$ ), a triglyceride concentration of $2,341 \mathrm{mg} \cdot \mathrm{dL}^{-1}$ and presence of chylomicrons was obtained. Microbiological and cytological results on pleural fluid were negative. A serum level of Ca 15.3 was normal.

Due to her previous diagnosis of breast carcinoma, we decided to perform PET before surgical exploration of the mediastinum and even before chest tube placement. Simultaneously, we prescribed oral fasting and parenteral nutrition. PET was normal; therefore, we opted for a conservative approach. Following thoracic radiographs, pleural effusion remained stable. Ten days later, it began to decrease slowly and finally disappeared after 3 weeks. Currently, 1 yr later, the patient remains asymptomatic and thoracic radiograph is normal.

Chylothorax occurs when the thoracic duct is disrupted and chyle enters the pleural space [3]. It is a relatively rare condition and represents $\sim 2 \%$ of all pleural effusions in adults [4]. A tumour is the leading cause in $>50 \%$ of cases, mainly the lymphoma group (which accounts for $75 \%$ ) and metastatic carcinoma. Surgical procedures account for most cases of traumatic chylothorax. Congenital and idiopathic chylothorax are the next leading causes; however, many idiopathic cases may be linked to an occult neoplasm after extensive evaluation or prolonged observation (6-12 months) [1]. Spontaneous chylothorax is a very infrequent cause of pleural effusion; a recent literature review reported only 13 cases had been described since 1960 [5]. It usually develops bilaterally, requires no aggressive therapeutic measures and no underlying pathology is apparent [4-6].

Establishing a cause is essential in nontraumatic chylothorax; CT studies of the mediastinum and even lymphangiogram are recommended. When CT examination reveals no lymphadenopathy or other masses and the chylothorax progresses, the patient should undergo videothoracoscopy or exploratory thoracotomy [1].

Metabolic or molecular imaging with fluorine-18 fluorodeoxy glucose PET has emerged as a powerful imaging modality for the diagnosis and staging of a variety of cancers, by far superior to CT [7], and its sensitivity and specificity is highest for supradiaphragmatic and mediastinal involvement [2]. To date, lymphoma and many types of carcinoma (including lung, breast and gynaecological cancer) are special indications for its use [8].

The use of positron emission tomography on evaluating a patient with chylothorax has not yet been reported in an extensive review of the literature in MEDLINE. In my opinion, positron emission tomography could play an excellent role in the management of nontraumatic chylothorax, mainly when the aetiology remains unknown after conventional procedures.

\section{A. Garcia-Zamalloa}

Hospital of Mendaro, Mendaro, Spain.

\section{REFERENCES}

1 Light RW. Chylothorax and Pseudochylothorax. In: Pleural Diseases. 4th Edn. Philadelphia, Lippincott Williams \& Wilkins, 2001; pp. 327-341.

2 Schiepers C, Filmont JE, Czernin J. PET for staging of Hodgkins and non-Hodgkins lymphoma. Eur J Nucl Med Mol Imaging 2003; 30: Suppl. 1, S82-S88.

3 Garcia-Zamalloa AM, Ruiz-Irastorza G, Aguayo FJ, Gurrutxaga N. Pseudochylothorax: report of 2 cases and review of the literature. Medicine (Baltimore) 1999; 78: 200-207.

4 Garcia Restoy E, Bella Cueto F, Espejo Arenas E, et al. Spontaneous bilateral chylothorax: uniform features of a rare condition. Eur Respir J 1988; 1: 872-873.

5 Bernier C, Haouzi S, Bouvard E, et al. Chylothorax spontané idiopathique. Revue de la littérature à propos dún cas [Spontaneous idiopathic chylothorax. Case report and review of the literature]. Rev Pneumol Clin 2000; 56: 369-373.

6 Gullane PJ, Marsh AS. Bilateral spontaneous chylothorax presenting as a neck mass. J Otolaryngol 1984; 13: 255-260.

7 Berman CG, Clark RA. Positron emission tomography in initial staging and diagnosis of persistent or recurrent disease. Curr Opin Oncol 2000; 12: 132-137.

8 Zimny M, Siggelkow W. Positron emission tomography scanning in gynaecologic and breast cancers. Curr Opin Obstet Gynecol 2003; 15: 69-75. 